\title{
A third-order extension to the Liénard oscillator and it's competitive modes analysis
}

\author{
Robert A. Van Gorder
}

Received: date / Accepted: date

\begin{abstract}
We study the dynamics of nonlinear differential equations of the form $\dddot{x}+f(x) \ddot{x}+g(x, \dot{x}) \dot{x}+$ $h(x)=0$, which is a third-order extension to the Liénard oscillator equation. This equation holds a number of interesting and physically relevant third-order dynamical systems as special cases. We present a general competitive modes analysis in order to derive some necessary conditions under which the such systems admit chaos. For several of the interesting reductions of the equations, we demonstrate that the approach allows us to determine parameter values and initial conditions which permit chaotic trajectories. We also demonstrate that, while competitive modes can be useful for finding chaotic regimes, the competitiveness conditions themselves are not a sufficient condition for chaos. In this way, we are able to discuss both the benefits and the limitations of the competitive modes approach. By doing this, we demonstrate that there are several reduction of this general third order equation which give chaos, including those of interest in theoretical physics and electrical engineering.
\end{abstract}

Keywords generalized Liénard oscillator · threedimensional nonlinear system $\cdot$ nonlinear dynamics . chaotic attractors $\cdot$ competitive modes

\section{Introduction}

The second order equation governing the Liénard oscillator [1] reads

$\ddot{x}+F(x) \dot{x}+G(x)=0$.

R. A. Van Gorder

Mathematical Institute, University of Oxford, Andrew Wiles Building, Radcliffe Observatory Quarter, Woodstock Road, Oxford OX2 6GG United Kingdom

E-mail: Robert.VanGorder@maths.ox.ac.uk
Equations of this type can be used to model oscillating circuits, and the Van der Pol oscillator is a special case. Mathematically, this equations and second order generalizations have been studied in order to generate a variety of oscillating and in some cases periodic solutions $[2,3]$. The problem with negative damping was considered in [4]. Solutions under one-sided growth restrictions were studied in [5]. Solutions with quadratic damping were studied in [6].

Consider the third order extension of the Liénard oscillator,

$\dddot{x}+f(x) \ddot{x}+g(x, \dot{x}) \dot{x}+h(x)=0$.

Note that while this seems somewhat arbitrary, it is a natural extension of (1). Indeed, the equation captures many of the properties of (1) in some reductions (as we shall later show), yet is of higher order, meaning that a variety of new dynamics should be possible. This thirdorder equation can be used to describe a number of physically relevant problems, as we shall demonstrate later. We may write (2) as the first order system

$$
\begin{aligned}
& \dot{y}_{1}=y_{2}, \\
& \dot{y}_{2}=y_{3}, \\
& \dot{y}_{3}=-f\left(y_{1}\right) y_{3}-g\left(y_{1}, y_{2}\right) y_{2}-h\left(y_{1}\right),
\end{aligned}
$$

which shall be useful in our later mathematical analysis. Note also that equilibrium points (steady states) of (2) correspond to $x^{*} \in \mathbb{R}$ such that $h\left(x^{*}\right)=0$, and hence equilibrium points of the system (3) will take the form $\left(y_{1}^{*}, y_{2}^{*}, y_{3}^{*}\right)=\left(x^{*}, 0,0\right)$ for $\operatorname{such} x^{*}$.

Sprott [7] studied a sub-class of equation (2) of the form $f(x)=A, g(x, \dot{x})=B$, where $A$ and $B$ are positive real-valued constants, that is,

$\dddot{x}+A \ddot{x}+B \dot{x}+h(x)=0$. 
As discussed in [7], equation (4) is essentially a damped harmonic oscillator driven by a linear memory term that involves the integral of $h(x)$. For many specific forms of $h(x)$, chaos was observed, both in the paper [7] and by others. Chaos was observed when $h(x)$ takes the form of a cubic function [8], while piecewise linear forms of $h(x)$ have also been shown [9-11] to yield chaos in (4). An RLC circuit has also been devised to give chaos for particular forms of $h(x)$, see $[12,13]$. Since (2) is therefore a generalization of the Sprott systems, we anticipate that chaotic dynamics will emerge from many equations of the form (2).

The goal of this paper is to investigate the nonlinear dynamics emergent in general equations of the form (2). In Section 2 we give a general competitive modes analysis for (3), in order to determine forms of (3) which might permit chaotic solutions. In Section 3, we consider three concrete physical applications (Sprott equations, electronic oscillator equations, memristor oscillators) which can be described by systems of the form (3). By way of these applications, we demonstrate the utility of considering systems of the form (3), as well as the utility of the competitive modes analysis of Section 2. In Section 4 we consider a specific form of (2) which satisfies the competitive modes conditions yet which nonetheless cannot yield chaotic dynamics. This illustrates the fact that the competitiveness conditions are not sufficient for the existence of chaotic trajectories in nonlinear systems. Concluding remarks are given in Section 5.

\section{Analytical considerations for (2)}

Recall that the method of competitive modes involves recasting a dynamical system as a coupled system of oscillators [14-19]. Consider the general nonlinear autonomous system of dimension $n$ given by

$\dot{x}_{i}=f_{i}\left(x_{1}, x_{2}, \ldots, x_{n}\right)$.

Differentiation of (5) once gives a coupled system of second order equations,

$$
\begin{aligned}
\ddot{x}_{i}=\sum_{j=1}^{n} f_{j} \frac{\partial f_{i}}{\partial x_{j}}= & -x_{i} g_{i}\left(x_{1}, x_{2}, \ldots, x_{i}, \ldots, x_{n}\right) \\
& +h_{i}\left(x_{1}, x_{2}, \ldots, x_{i-1}, x_{i+1}, \ldots, x_{n}\right) .
\end{aligned}
$$

When a $g_{i}$ is positive, its respective $i$ th equation behaves like an oscillator. The following conjecture is posed in $[15]$ :

Competitive Modes Requirements: The conditions for dynamical systems to be chaotic are given by:

(A) there exist at least two modes, labeled $g_{i}$ in the system;

(B) at least two $g$ 's are competitive or nearly competitive, that is, for some $i$ and $j, g_{i} \approx g_{j}>0$ at some $t$;

(C) at least one of the $g$ 's is a function of evolution variables such as $t$; and

(D) at least one of the $h$ 's is a function of system variables.

The requirements (A)-(D) essentially tell us that a condition for chaos is that two or more equations in (6) behave as oscillators $\left(g_{i}>0\right)$, and that two of these oscillators lock frequencies at one or more times. In practice, we find that the frequencies agree at a countably infinite collection of time values $[14,19]$. The frequencies should be functions of time (i.e., we have nonlinear frequencies), and there should be at least one forcing function which depends on a state variable.

Returning to the equations of interest, differentiation of (3) once yields

$$
\begin{aligned}
& \ddot{y}_{1}=H_{1}\left(y_{3}\right), \\
& \ddot{y}_{2}+G_{2}\left(y_{1}, y_{2}\right) y_{2}=H_{2}\left(y_{1}, y_{3}\right), \\
& \ddot{y}_{3}+G_{3}\left(y_{1}, y_{2}\right) y_{3}=H_{3}\left(y_{1}, y_{2}\right),
\end{aligned}
$$

where $H_{1}\left(y_{3}\right)=y_{3}, H_{2}\left(y_{1}, y_{3}\right)=-f\left(y_{1}\right) y_{3}-h\left(y_{1}\right)$, $H_{3}\left(y_{1}, y_{3}\right)=-g_{1}\left(y_{1}, y_{2}\right) y_{2}^{2}-h^{\prime}\left(y_{1}\right) y_{2}+f\left(y_{1}\right) g\left(y_{1}, y_{2}\right) y_{2}+$ $f\left(y_{1}\right) h\left(y_{1}\right), G_{1}=0, G_{2}\left(y_{1}, y_{2}\right)=g\left(y_{1}, y_{2}\right), G_{3}\left(y_{1}, y_{2}\right)=$ $f^{\prime}\left(y_{1}\right) y_{2}+g_{2}\left(y_{1}, y_{2}\right) y_{2}+g\left(y_{1}, y_{2}\right)-\left(f\left(y_{1}\right)\right)^{2}$. Note that we define the partial derivative notation $\frac{\partial g}{\partial y_{1}}=g_{1}$ and $\frac{\partial g}{\partial y_{2}}=g_{2}$.

Since the equation for $y_{1}$ can never be an oscillator equation, hence $G_{1}=0$, only the modes $y_{2}$ and $y_{3}$ can ever be competitive. Therefore, consider the case when both modes are competitive, that is, when the mode frequencies satisfy $G_{2}\left(y_{1}, y_{2}\right)=G_{3}\left(y_{1}, y_{2}\right)$. Then, we have that $y_{1}$ and $y_{2}$ must satisfy

$f^{\prime}\left(y_{1}\right) y_{2}+g_{2}\left(y_{1}, y_{2}\right) y_{2}-\left(f\left(y_{1}\right)\right)^{2}=0$.

Therefore, if condition (8) holds at a point $t=t_{0} \geq 0$, then the modes $y_{2}$ and $y_{3}$ are competitive at $t=t_{0}$.

\section{$2.1 \mathrm{~g}$ constant in $y_{2}$}

In the case where $g$ is constant in $y_{2}$, that is $g=g\left(y_{1}\right)$, we must have that $G_{2}=g\left(y_{1}\right)>0$ and $G_{3}=f^{\prime}\left(y_{1}\right) y_{2}+$ $g\left(y_{1}\right)-\left(f\left(y_{1}\right)\right)^{2}>0$. The competitiveness condition (8) becomes

$f^{\prime}\left(y_{1}\right) y_{2}-\left(f\left(y_{1}\right)\right)^{2}=0$ 
SO

$y_{2}=\left(f\left(y_{1}\right)\right)^{2} / f^{\prime}\left(y_{1}\right)$

is the explicit form of the competitiveness condition. This suggest that ODEs of the form

$\dddot{x}+f(x) \ddot{x}+g(x) \dot{x}+h(x)=0$

can admit chaotic behaviors if $g\left(x\left(t_{0}\right)\right)>0$ and $\dot{x}\left(t_{0}\right)=$ $\left(f\left(x\left(t_{0}\right)\right)\right)^{2} / f^{\prime}\left(x\left(t_{0}\right)\right)$ at some $t=t_{0}$.

Note that if $f^{\prime}\left(x\left(t_{0}\right)\right)=0$, the modes can still be competitive. The conditions required in this case are $g\left(x\left(t_{0}\right)\right)>0$ and $f\left(x\left(t_{0}\right)\right)=0$.

$2.2 g\left(y_{1}, y_{2}\right)=k\left(y_{1}\right)+l\left(y_{1}\right) y_{2}$

In the case where $g$ depends linearly on $y_{2}$, such as $g\left(y_{1}, y_{2}\right)=k\left(y_{1}\right)+l\left(y_{1}\right) y_{2}$, we must have the conditions $G_{2}=k\left(y_{1}\right)+l\left(y_{1}\right) y_{2}>0$ and $G_{3}=f^{\prime}\left(y_{1}\right) y_{2}+k\left(y_{1}\right)+$ $2 l\left(y_{1}\right) y_{2}-\left(f\left(y_{1}\right)\right)^{2}>0$. Condition (8) then becomes

$f^{\prime}\left(y_{1}\right) y_{2}+l\left(y_{1}\right) y_{2}-\left(f\left(y_{1}\right)\right)^{2}=0$.

The modes $y_{2}$ and $y_{3}$ are then competitive at $t=t_{0}$ provided that

$$
\begin{aligned}
k\left(y_{1}\left(t_{0}\right)\right)+l\left(y_{1}\left(t_{0}\right)\right) y_{2}\left(t_{0}\right)>0 & \\
f^{\prime}\left(y_{1}\left(t_{0}\right)\right) y_{2}\left(t_{0}\right)+k\left(y_{1}\left(t_{0}\right)\right)+ & 2 l\left(y_{1}\left(t_{0}\right)\right) y_{2}\left(t_{0}\right) \\
& -\left(f\left(y_{1}\left(t_{0}\right)\right)\right)^{2}>0
\end{aligned}
$$

and

$y_{2}\left(t_{0}\right)=\frac{f\left(y_{1}\left(t_{0}\right)\right)^{2}}{f^{\prime}\left(y_{1}\left(t_{0}\right)\right)+l\left(y_{1}\left(t_{0}\right)\right)}$.

Performing some simplifications, this suggests that third order ODEs of the form

$\dddot{x}+f(x) \ddot{x}+k(x) \dot{x}+l(x) \dot{x}^{2}+h(x)=0$

can admit chaos if

$\dot{x}\left(t_{0}\right)=\frac{f\left(x\left(t_{0}\right)\right)^{2}}{f^{\prime}\left(x\left(t_{0}\right)\right)+l\left(x\left(t_{0}\right)\right)}$

and

$k\left(x\left(t_{0}\right)\right)+\frac{l\left(x\left(t_{0}\right)\right) f\left(x\left(t_{0}\right)\right)^{2}}{f^{\prime}\left(x\left(t_{0}\right)\right)+l\left(x\left(t_{0}\right)\right)}>0$,

at some $t=t_{0}$.

We should remark that, if $f^{\prime}\left(x\left(t_{0}\right)\right)+l\left(x\left(t_{0}\right)\right)=0$, the modes can still be competitive. The conditions for this case become $k\left(x\left(t_{0}\right)\right)>0$ and $f\left(x\left(t_{0}\right)\right)=0$.
2.3 The zero locus of $(8)$

In general, we cannot find a closed form expression for $y_{2}$ in terms of $y_{1}$ from (8) when $g\left(y_{1}, y_{2}\right)$ is a general nonlinear function. However, one can study the zero locus which constitutes the solution set of ordered pairs $\left(y_{1}, y_{2}\right)$ to $(8)$.

Note also that, since only two modes can ever be competitive, we only have one relation to check (namely, (8)), along with the positivity conditions. In general, for a third-order system, we would have three possible comparisons to make. This simplicity is due to the form of the equation (2) considered.

\subsection{Volume expansion or contraction}

In addition to competitive modes, one can use volume expansion or contraction in an attempt to better understand the dynamics of specific equations of the form (2). Note that the system (3) has volume expansion or contraction in phase space depending on the sign of

$\frac{1}{\mathcal{V}} \frac{d \mathcal{V}}{d t}=\Delta \mathcal{V}=\frac{\partial \dot{y}_{1}}{\partial y_{1}}+\frac{\partial \dot{y}_{2}}{\partial y_{2}}+\frac{\partial \dot{y}_{3}}{\partial y_{3}}=-f\left(y_{1}\right)=-f(x)$

In particular, for a chaotic attractor, we should have

$0 \geq \frac{1}{\mathcal{V}} \frac{d \mathcal{V}}{d t}=-f(x)$

for long time if we want trajectories in phase space to converge upon such an attractor. For more on dissipitivity and the utility of this approach for locating hidden attractors, see [21-24].

In light of $(20), f(x) \geq 0$ seems to be a useful condition on equations of the form (2) when we seek chaos. For some situations, such as the blue skye catastrophe [25], there is volume expansion locally for some time during bursting events, while for most times there is strong volume contraction. For such a case, a restriction that

$\int_{t_{0}}^{t_{f}} f(x(t)) d t \geq 0$

for every large enough time interval $\left[t_{0}, t_{f}\right]$, may be most useful. This says that, over every large enough time interval, the dynamics are dissipative, although there can be regions of local bursting behavior. As we shall see later in Section 3, the physical models which occur as special cases of (2) will satisfy this property. 


\section{Concrete examples}

In this section, we shall provide some examples of nonlinear oscillator equations of the form (2). We demonstrate that some equations of this form can permit chaos, and hence the extension of (1) to order three gives the possibility for many more types of dynamics.

\subsection{Sprott equations}

Sprott [7] considered various equations of the form

$\dddot{x}+a \ddot{x}+b \dot{x}+h(x)=0$,

and some specific functional forms of $h(x)$ for which chaotic trajectories exist were found. Such equations have volume contraction when $a>0$ or are volume neutral when $a=0$.

The mode frequencies for (22) are found to be $G_{1}=$ $0, G_{2}=b$, and $G_{3}=b-a^{2}$. Since the mode frequencies are all constant, if $G_{2}=G_{3}$ then the mode frequencies are always competitive. In this case, the modes lock and the system may not exhibit chaos for some response functions $h(x)$ (see [7]). However, if the mode frequencies are nearly competitive, $G_{2} \approx G_{3}$, then chaos can still occur. This would imply that we should have the condition $0 \leq a^{2}<1$, and this is actually what we see in all of the chaotic systems of the form (22) given in Table 1 of [7]. From the positivity conditions on $G_{2}$ and $G_{3}$, we must also have $b-a^{2}>0$. Therefore, it makes sense to look for chaos in the system (22) when

$a^{2}<<1$ and $a^{2}<b$.

When $h(x)$ is a linear function, note that the equation (22) can never give chaos, hence a competitive modes analysis is not sufficient for chaos. We shall pick various nonlinear forms of $h(x)$. In particular, we take $h(x)=x-x^{2}, h(x)=x-2 \tanh (x)$, and $h(x)=$ $-\sin (x)$, and plot the resulting phase portraits in Figs. 1,2 , and 3 , respectively. For each case, we pick parameter values giving chaos, using the results of [7] as guidance. For each case, one positive, one zero, and one negative Lyapunov exponent exists. Note that the modes are competitive or nearly competitive for each, while the positivity conditions always hold. Therefore, even through the competitiveness and positivity conditions are independent of time (and hence hold for all time, when appropriate parameter values are employed), we still see emergent chaotic behavior within the dynamics of equation (22).

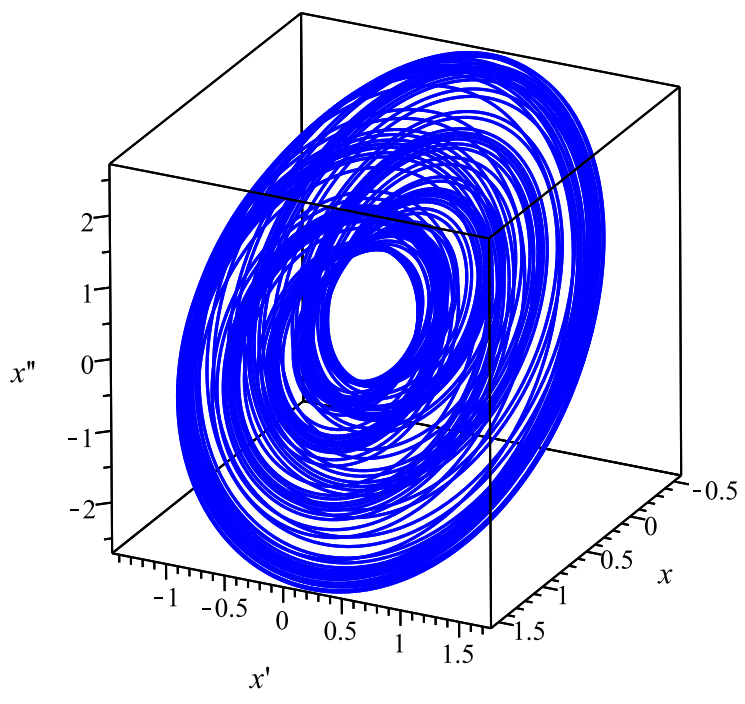

Fig. 1 Plot of the phase portrait for the system (22) when $a=0, b=2.8$, and $h(x)=x-x^{2}$. Initial conditions are taken to be $x(0)=0.5, x^{\prime}(0)=-1$, and $x^{\prime \prime}(0)=1$. Since $a=0$, the modes are always competitive, while since $b=1>a^{2}$ the positivity condition is always satisfied.

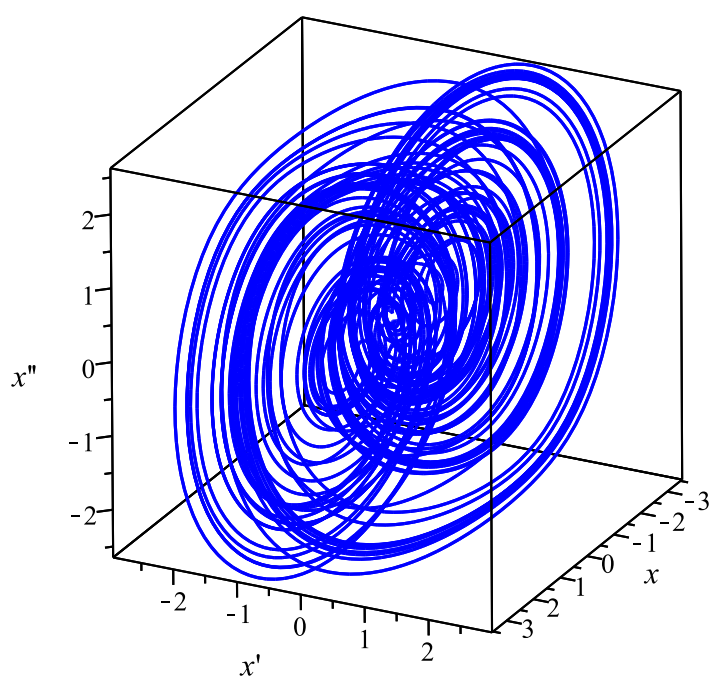

Fig. 2 Plot of the phase portrait for the system (22) when $a=0.19, b=1$, and $h(x)=x-2 \tanh (x)$. Initial conditions are taken to be $x(0)=0, x^{\prime}(0)=1$, and $x^{\prime \prime}(0)=0$. Since $a^{2}=0.0361<<1$, the modes are always nearly competitive, while since $b=1>a^{2}$ the positivity condition is always satisfied.

\subsection{Electronic oscillator}

In [26], a simple chaotic oscillator from an electric circuit was constructed. This oscillator was modeled by the ODE system

$$
\begin{aligned}
\dot{X} & =Y, \\
\dot{Y} & =a Y-X-Z, \\
\epsilon \dot{Z} & =b+Y-c\left(e^{Z}-1\right),
\end{aligned}
$$




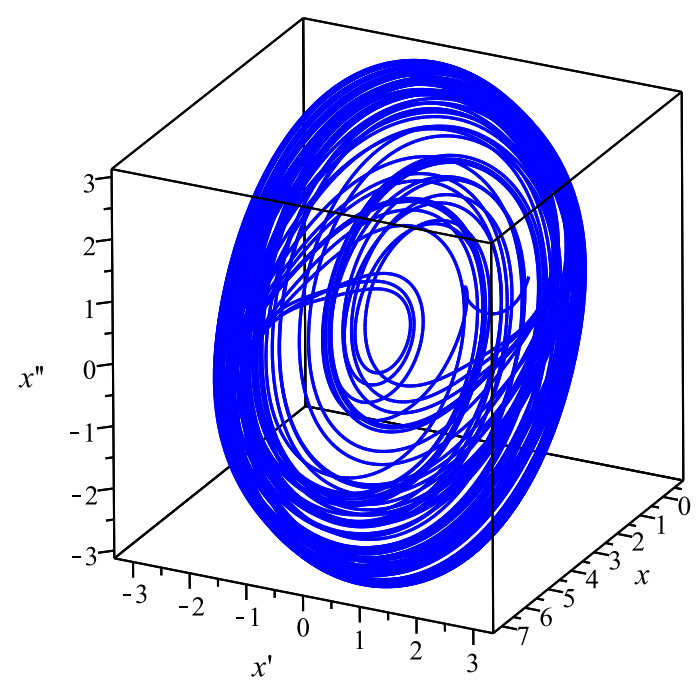

Fig. 3 Plot of the phase portrait for the system (22) when $a=0.2, b=1$, and $h(x)=-\sin (x)$. Initial conditions are taken to be $x(0)=0, x^{\prime}(0)=1$, and $x^{\prime \prime}(0)=0$. Since $a^{2}=$ $0.04<<1$, the modes are always nearly competitive, while since $b=1>a^{2}$ the positivity condition is always satisfied.

where $a, b, c, \epsilon$ are constant parameters. Inportantly, chaotic dynamics were shown to exist for such oscillators in [26].

In our coordinates, the relevant oscillator equation is given by

$$
\dddot{x}+\left(C e^{x}-A\right) \ddot{x}+\left(C e^{x} \dot{x}+D-A e^{x}\right) \dot{x}+\left(C e^{x}-B\right)=0 .
$$

Our function $x(t)$ is $z(t)$ in [26], while the parameters $a, b, c, \epsilon$ of $[26]$ are transformed by $A=\frac{a c}{\epsilon}, B=\frac{b+c}{\epsilon}$, $C=\frac{c}{\epsilon}, D=\frac{1}{\epsilon}+1$. Note that $x=\ln (B / C)$ is an equilibrium value. We have volume contraction in phase space when $C e^{x}-A>0$.

We find that the positivity conditions are given by

$(C \dot{x}-A) e^{x}+D>0$

and

$(3 C \dot{x}+2 A C-A) e^{x}-C^{2} e^{2 x}-A^{2}>0$.

The competitiveness condition becomes

$2 C e^{x} \dot{x}-\left(C e^{x}-A\right)^{2}=0$,

Hence, if there exists $t=t_{0}$ such that (28) holds, then the mode frequencies $G_{2}$ and $G_{3}$ are equal. In terms of the unknown functions, the competitiveness condition is equivalent to

$\dot{x}\left(t_{0}\right)=\frac{C}{2} e^{x\left(t_{0}\right)}-A+\frac{A^{2}}{2 C} e^{-x\left(t_{0}\right)}$.

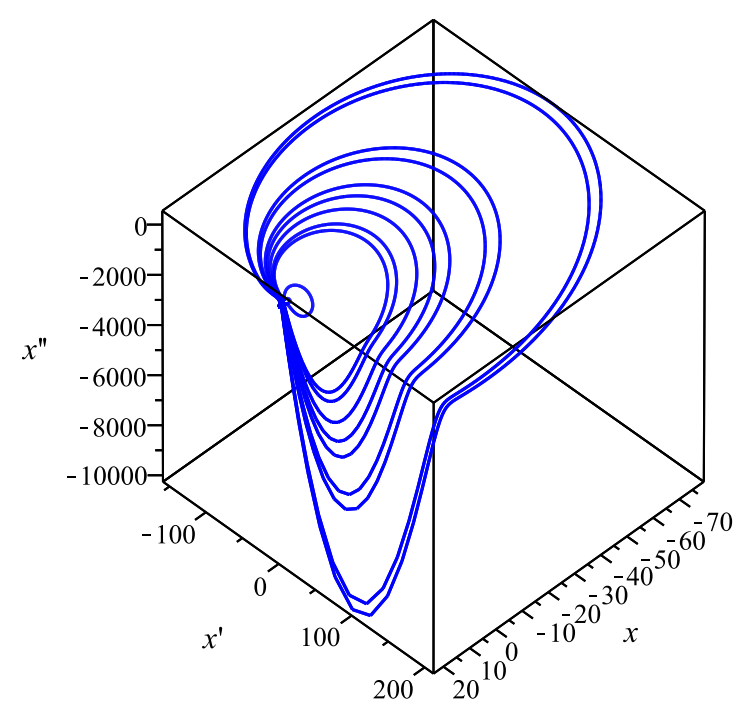

Fig. 4 Plot of the phase portrait for the system (25) when $a=0.4, b=30, c=4 \times 10^{-9}$, and $\epsilon=0.13$, which in turn give $A=1.2307 \times 10^{-9}, B=230.769, C=3.0769 \times 10^{-8}$, and $D=8.6923$. Initial conditions are taken to be $x(0)=1$, $x^{\prime}(0)=0$, and $x^{\prime \prime}(0)=0$. The corresponding plot showing the competitiveness condition is given in Fig. 5 .

If we use the competitiveness condition in the positivity condition, we find that (after some algebraic manipulations) they may be reduced to a single relation of the form

$\frac{1}{2} C^{2} e^{2 x}-A(C+1) e^{x}+\frac{1}{2} A^{2}>\max \{0,-D\}$.

Note that the parameter $B$ does not matter for either positivity or competitiveness, while $D$ enters only into the positivity condition. In contrast, both $A$ and $C$ feature in both the positivity and competitiveness conditions, and are hence more likely to play the role of bifurcation parameters.

We plot a representative chaotic solution in Fig. 4 for fixed parameter values. In Fig. 5, we plot the competitiveness condition (28) as well as the positivity condition (30) corresponding to this chaotic solution. We see that the competitiveness condition holds intermittently over the time domain, which appears as a common feature among chaotic systems [27]. Therefore, the competitive modes analysis can be successfully applied to the solutions of (25).

\subsection{Memristor oscillator}

Itoh and Chua [28] studied a variety of nonlinear dynamics arising from memristor oscillators. We shall con- 


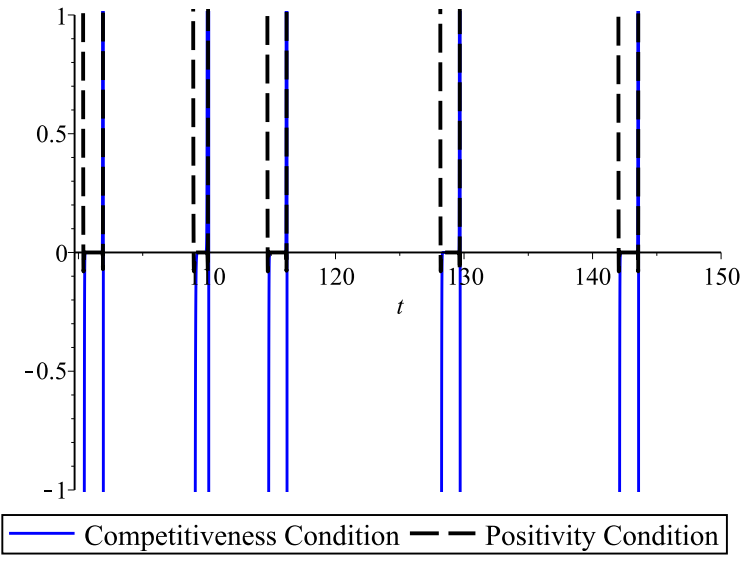

Fig. 5 Plot of the competitiveness and positivity conditions for equation (25), given parameter values and initial conditions of Fig. 4. When the competitiveness condition equals zero and the function showing the positivity condition is greater than zero, then the criteria for the competitive modes test are met, and the two relevant modes are indeed competitive. This occurs at what appears to be countably many intermittent times.

sider the specific third order system

$$
\begin{aligned}
\dot{X} & =\alpha(Y-W(Z) X), \\
\dot{Y} & =-\xi X+\beta Y, \\
\dot{Z} & =X,
\end{aligned}
$$

where $W(Z)$ is a prescribed function. This equation arises in the study of memristors, and appears as equation (65) of [28]. The functional form of $W$ will determine the particular physics of the system.

Putting this equation into the form of a single ODE by reduction of order [29], and setting $x(t)=Z(t)$, we obtain

$\dddot{x}+(\alpha W(x)-\beta) \ddot{x}+\alpha\left(W^{\prime}(x) \dot{x}-\beta W(x)+\xi\right) \dot{x}=0$.

We have $f(x)=\alpha W(x)-\beta$ and $g(x, \dot{x})=\alpha\left(W^{\prime}(x) \dot{x}-\right.$ $\beta W(x)+\xi)$. We have volume contraction in phase space provided that $\alpha W(x)-\beta>0$.

The competitiveness condition is

$2 \alpha W^{\prime}(x) \dot{x}-(\alpha W(x)-\beta)^{2}=0$,

and for $W^{\prime}(x) \neq 0$ this gives

$\dot{x}\left(t_{0}\right)=\frac{\left(\alpha W\left(x\left(t_{0}\right)\right)-\beta\right)^{2}}{2 \alpha W^{\prime}\left(x\left(t_{0}\right)\right)}$.

Meanwhile, the positivity condition becomes

$\alpha\left(W^{\prime}(x) \dot{x}-W(x)+\xi\right)>0$,

which is equivalent to (using (34))

$\frac{\alpha^{2}}{2}(W(x))^{2}-\alpha(1+\beta) W(x)+\frac{\beta^{2}}{2}+\alpha \xi>0$.

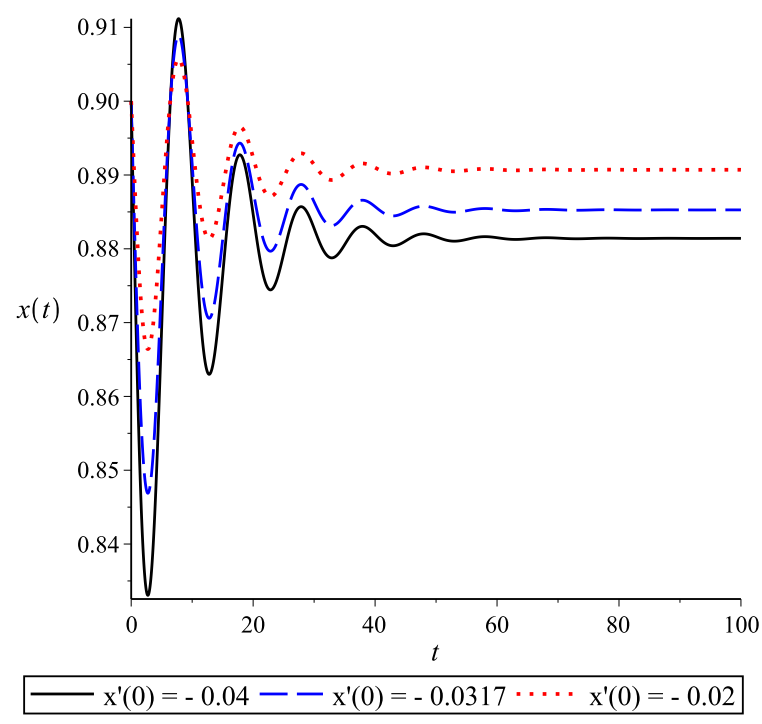

Fig. 6 Plot of the solution $x(t)$ to (32) given $\alpha=0.2, \beta=0$, $\xi=2$ while $W(x)$ is taken as in (38) with parameters $a=0$, $b=1, j=20, k=1$. Initial conditions are $x(0)=0.9$, $x^{\prime \prime}(0)=0$, while $x^{\prime}(0)$ is varied. When $x^{\prime}(0)=-0.0317$ we have from (34) that the modes are competitive.

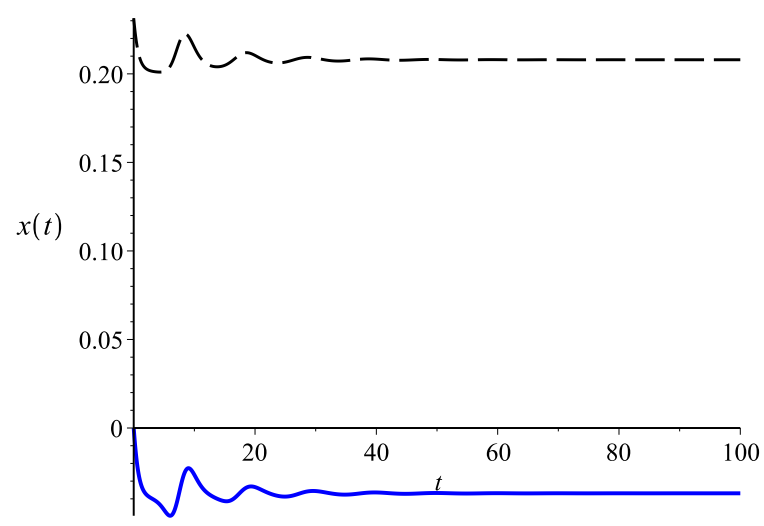

Competitiveness Condition -- Positivity Condition

Fig. 7 Plot of the competitiveness (33) and positivity (35) conditions for equation (32), given parameter values and initial conditions of Fig. 6. We select $x^{\prime}(0)=-0.0317$, noting similar results for nearby initial conditions. Observe that the positivity condition always holds, while the competitiveness condition holds only at $t=0$, and not for any positive time. Chaos does not emerge, which highlights the need for modes to be intermittently competitive for all time if chaotic trajectories are sought.

On the other hand, if $W^{\prime}\left(x\left(t_{0}\right)\right)=0$, we must have the competitiveness condition $W\left(x\left(t_{0}\right)\right)=\frac{\beta}{\alpha}$. The positivity condition is then $\xi>\frac{\beta}{\alpha}$.

In [28], $W(x)$ was taken to be of the form of a family of step functions. For our purposes, consider a family of step functions satisfying

$W(x)= \begin{cases}a & \text { if }|x-k|<1 \\ b & \text { if }|x-k|>1\end{cases}$ 
Since this function is discontinuous, let us approximate it via a continuous and smooth function. To this end, consider

$W(x)=a+(b-a) \tanh ^{2}(j(x-k))$,

which for large $j>>1$ gives a smooth approximation of (37).

In Fig. 6 we plot the resulting solution $x(t)$ to $(32)$ for various values of the initial condition near the algebraic competitiveness condition, corresponding to $x^{\prime}(0)=$ -0.0317 when $x(0)=0.9$. We find that the trajectories tend to a stable positive equilibrium. For other parameters, stable limit cycles exist (as discussed in [28]). Therefore, while we have competitiveness of two modes, we do not have chaos.

Let us consider the positivity condition (35) and the competitiveness condition (33). We plot both in Fig. 7, for parameter values and initial conditions taken in Fig. 6 . Initially the modes are competitive, but then fail to be competitive for all time $t>0$. The positivity condition is satisfied for all $t>0$. This highlights the fact that the modes should be intermittently competitive in order for there to be chaos, while modes which are competitive only at finitely many times do not yield chaotic dynamics (as was discussed in [27]). As such, it is insufficient for modes to be competitive at only a point (or, by extension, a finite collection of points) if we seek chaotic trajectories.

We remark that the competitive modes analysis has picked up on something interesting. Since the modes are competitive at only one time, this means that as time gets arbitrarily large, the modes will never again be competitive. (Similarly, if the modes are competitive at finitely many times, then for all times larger than the maximum of those finite times, the modes will not be competitive.) As such, we anticipate rather tame behaviors: trajectories leading to finite steady states, divergence to infinity, or limit cycles. That is, we essentially expect those dynamics commonly seen in two dimensions. With this in mind, let us note that equations of the form (32) with sufficiently smooth $W(x)$ can actually be written in the form

$\frac{d}{d t}\{\ddot{x}+\alpha W(x) \dot{x}-\beta \dot{x}+\alpha W(x)+\alpha \xi x\}=0$,

and hence for such a case, equation (32) clearly has a first integral for the form

$\ddot{x}+\alpha W(x) \dot{x}-\beta \dot{x}+\alpha W(x)+\alpha \xi x=I$,

where $I$ is a real-valued constant of integration. Since this is a second order equation, and the nonlinear terms are smooth, we can only obtain non-chaotic solutions. Hence, even if modes start out competitive, they should

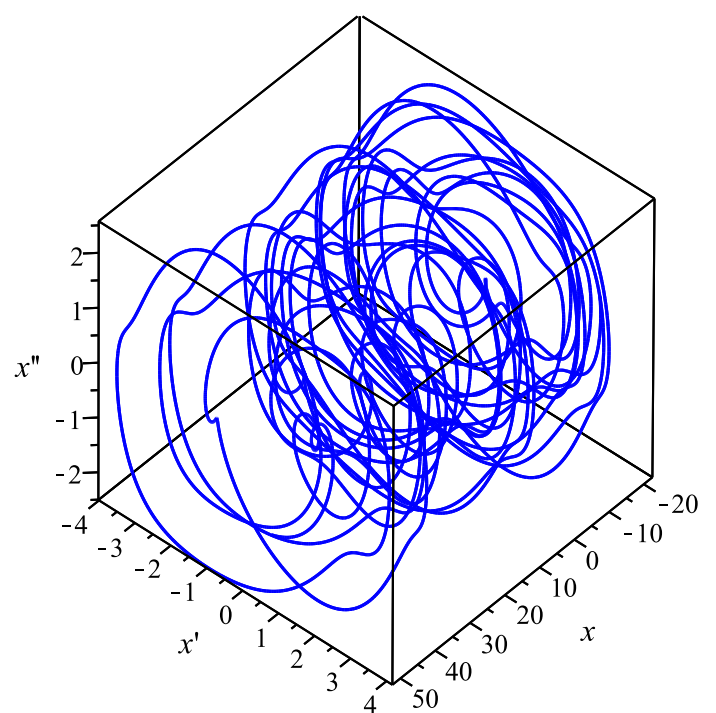

Fig. 8 Plot of the phase portrait for the system to (42) given $\alpha=0.2, \beta=0, \xi=2$ while $W(x)$ is taken as in (38) with parameters $a=0, b=1, j=20, k=1$. We also take $V(x)=$ $5 \sin (x)$ for sake of example. Initial conditions are $x(0)=0.9$, $x^{\prime}(0)=-0.0317$, and $x^{\prime \prime}(0)=0$. Recall that when $x^{\prime}(0)=$ -0.0317 , we have from (34) that the modes are competitive.

separate, with one mode eventually becoming dominant for increasingly large time.

Let us note that one can modify the oscillator equation (31) in order to obtain chaotic trajectories. We consider the system

$$
\begin{aligned}
\dot{X} & =\alpha(Y-W(Z) X), \\
\dot{Y} & =-\xi X+\beta Y-V(Z), \\
\dot{Z} & =X,
\end{aligned}
$$

where we now have coupled the $\dot{Y}$ equation with $Z$ direction, through use of the function $V(Z)$. Then, again identifying $x(t)=Z(t)$, we obtain the analogue of (32), to wit:

$\dddot{x}+(\alpha W(x)-\beta) \ddot{x}+\alpha\left(W^{\prime}(x) \dot{x}-\beta W(x)+\xi\right) \dot{x}+\alpha V(x)=0$.

Recall that the function $h(x)$ in (2) will not enter into either the competitiveness or positivity conditions, therefore we can use the same conditions as derived above. In Fig. 8, we plot a solution to (42) for which the modes are competitive at $t=0$. This solution corresponds to the choice $V(x)=5 \sin (x)$. In Fig. 9 we plot the competitiveness condition along with the positivity condition. As we can see, there are intermittent regions where modes are not competitive, while for most times the modes are indeed competitive. For large time, the competitiveness condition is nearly, but not exactly, zero, and the modes appear to remain nearly competitive for 


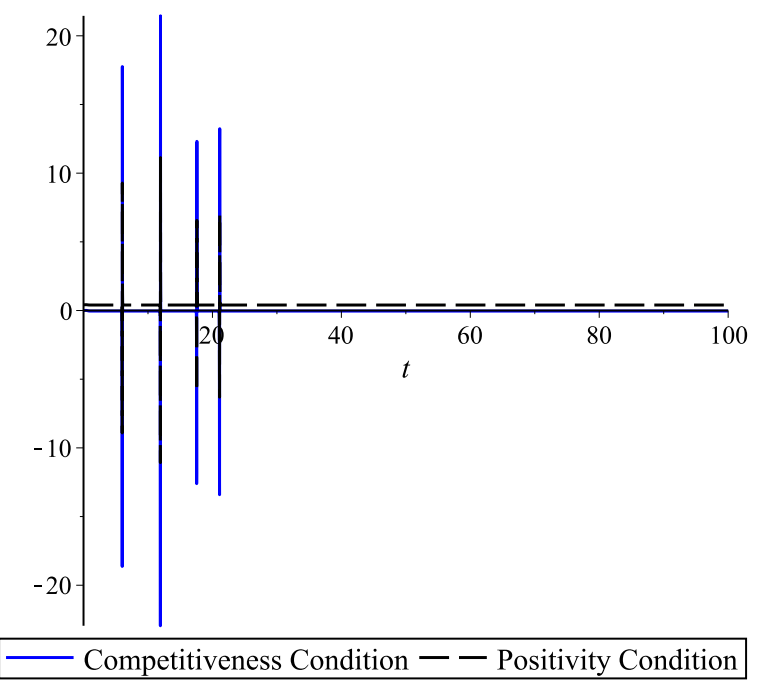

Fig. 9 Plot of the competitiveness (33) and positivity (35) conditions for equation (32), given parameter values and initial conditions of Fig. 8. We select $x^{\prime}(0)=-0.0317$, noting similar results for nearby initial conditions. Observe that the positivity condition holds over most of the domain, including for all sufficently large times, while the competitiveness condition holds at $t=0$ and for a collection of larger times. Eventually, the modes become locked at a nearly competitive state, with the curve denoting the competitiveness condition tending toward a value of about -0.04 for large time, which is sufficiently close to zero. As such, we observe the chaos as seen in Fig. 8.

all time. Meanwhile, the positivity condition is simultaneously satisfied for large time.

\section{Competitive modes are not sufficient for chaos}

While we demonstrated certain conditions for which two modes being competitive did not yield chaos (mainly, when two modes were competitive at only finitely many points), in this section we shall more generally consider the insufficiency of competitive modes, as illustrated by a subclass of equations of the form (2).

When (2) is equivalent to (1), we do not expect chaos, since in this case the dynamics are two-dimensional. Differentiation of (1) with respect to $t$ yields a third order system

$\dddot{x}+F(x) \ddot{x}+F^{\prime}(x) \dot{x}^{2}+G^{\prime}(x) \dot{x}=0$.

Equations (2) and (1) are therefore equivalent when $f(x)=F(x), g(x, \dot{x})=F^{\prime}(x) \dot{x}+G^{\prime}(x)$ and $h(x)=0$. In other words, when $h(x)=0$ and when $g$ takes the form $g(x, \dot{x})=f^{\prime}(x) \dot{x}+g_{0}(x)$, (2) has a first integral and hence it's dynamics are two-dimensional.
For a concrete example, consider the third-order nonlinear differential equation

$\dddot{x}+x \ddot{x}+\dot{x}^{2}+\left(\frac{1}{2} x^{2}+1\right) \dot{x}=0$.

The competitiveness condition is

$\dot{x}-\frac{1}{2} x^{2}=0$,

while the positivity condition is

$x^{2}+1>0$,

which always holds. Let us take $t_{0}=0$ to be a point when the modes are competitive. Then the mode frequencies $G_{2}=G_{3}$ at $t=0$ provided that $\dot{x}(0)=\frac{1}{2} x(0)^{2}$. Therefore, (44) has two competitive modes at time $t=$ 0 provided that the initial condition is selected appropriately.

While (44) has two competitive modes at time $t=0$, we can show that the system (44) cannot admit any chaotic trajectories. Note that (44) is equivalent to

$\frac{d}{d t}\left\{\ddot{x}+x \dot{x}+\frac{1}{6} x^{3}+x\right\}=0$.

Yet, (47) has an exact first integral, given by

$\ddot{x}+x \dot{x}+\frac{1}{6} x^{3}+x=C$.

The dynamics of (48) are planar, therefore there can be no chaotic trajectories. Yet, (44) and (48) are equivalent systems, so there can be no chaotic trajectory in (44). This demonstrates that the competitiveness of two modes cannot be a sufficient condition for chaos in dynamical systems of order greater than two.

Of course, this does not diminish the utility of the competitive modes analysis. The method remains useful for detecting parameter regimes that might lead to chaos. However, there still is a certain skill required to extract such information from the method. In this way, we see that the approach is certainly not a black box method, and that one must exercise caution when applying the method. Still, the approach has been demonstrated to assist in finding parameter regimes admitting chaos, for appropriate applications of the method.

\section{Conclusions}

A new, third-order, extension of the Liénard oscillator equation (2) was considered. A general competitive modes analysis for this third-order system was considered, as nonlinear third-order systems can possibly give chaos. This equation holds the third order equations 
studied in [7] as special cases, and hence we are able to study chaos in a much more general setting. Indeed, through a competitive modes analysis, we were able to derive some criteria which allow one to search for chaos in such third order systems. The results demonstrate that the natural extension (2) of the Liénard oscillator equation to third order permits a variety of dynamics, some previously explored for specific forms of (2).

Physically relevant examples were considered, in order to illustrate the utility of studying such third-order oscillator equations. In each of these cases, the aforementioned competitive modes analysis was employed in order to search for parameter regimes permitting the existence of chaotic trajectories. The results also point out that modes should be at least intermittently competitive over the time domain. When modes are competitive or nearly competitive at a single point (or a finite collection of points) in time, the dynamics observed are non-chaotic.

Non-sufficiency of competitive modes analysis was demonstrated for a specific reduction of (2). In particular, one may have modes which are competitive or nearly competitive, even when planar dynamics are observed. This means that the competitive modes analysis can be used as a diagnostic tool for trying to identify parameter regimes and initial conditions which permit chaotic trajectories in phase space, yet cannot be said to be sufficient conditions for the existence of such chaotic trajectories. Indeed, the existence of two competitive modes may be necessary (as conjectured elsewhere), although as we show here certainly is not sufficient, for the existence of chaotic trajectories in autonomous, continuous nonlinear systems of dimension three or greater.

\section{References}

1. A. Liénard, Etude des oscillations entretenues, Revue génrale de l'électricité 23, 901-912 (1928).

2. G. Villari, Periodic Solutions of Liénard's Equation, J. Math. Anal. Appl. 86, 379-386 (1982).

3. G. Villari, On the qualitative behaviour of solutions of Liénard equation, Journal of Differential Equations 67, 269-277 (1987).

4. J.R. Graef, On the generalized Liénard equation with negative damping, Journal of Differential Equations 12, 34-62 (1972).

5. P. Omari, G. Villari, and F. Zanolin, Periodic solutions of the Liénard equation with one-sided growth restrictions, Journal of Differential Equations 67, 278-293 (1987).

6. F. Dumortier and C. Li, Quadratic Liénard equations with quadratic damping, Journal of Differential Equations 139, 41-59 (1997).

7. J.C. Sprott, Simple chaotic systems and circuits, American Journal of Physics 68, 758-763 (2000).
8. P. Coullet, C. Tresser, and A. Arnéodo, Transition to stochasticity for a class of forced oscillators, Physics Letters A 72, 268-270 (1979).

9. A. Arneodo, P. Coullet, and C. Tresser, Possible new strange attractors with spiral structure, Communications in Mathematical Physics 79, 573-579 (1981).

10. A. Arneodo, P. Coullet, and C. Tresser, Oscillators with chaotic behavior: An illustration of a theorem by Shilnikov, Journal of Statistical Physics 27, 171-182 (1982).

11. L. O. Chua and F. Ayrom, Designing non-linear single op-amp circuits: A cookbook approach, International Journal of Circuit Theory and Applications 13, 235-268 (1985).

12. N. F. Rulkov, A. R. Volkovskii, A. Rodriguez-Lozano, E. Del Río, and M. G. Velarde, Mutual synchronization of chaotic self-oscillators with dissipative coupling, International Journal of Bifurcation Chaos 2, 669-676 (1992).

13. E. Del Río, M. G. Velarde, A. Rodríguez-Lozano, N. F. Rulkov, and A. R. Volkovskii, Experimental evidence for synchronous behavior of chaotic nonlinear oscillators with unidirectional or mutual driving, International Journal of Bifurcation Chaos 4, 1003-1009 (1994).

14. S. R. Choudhury and R. A. Van Gorder, Competitive modes as reliable predictors of chaos versus hyperchaos and as geometric mappings accurately delimiting attractors, Nonlinear Dynamics 69, 2255 (2012).

15. W. Yao, P. Yu, and C. Essex, Estimation of chaotic parameter regimes via generalized competitive mode approach, Communications in Nonlinear Science and Numerical Simulation 7, 197 (2002).

16. P. Yu, W. Yao, and G. Chen, Analysis on topological properties of the Lorenz and the Chen attractors using GCM, International Journal of Bifurcation and Chaos 17, 2791 (2007).

17. Z. Chen, Z.Q. Wu, and P. Yu, The critical phenomena in a hysteretic model due to the interaction between hysteretic damping and external force, Journal of Sound and Vibration 284, 783 (2005).

18. W. Yao, P. Yu, C. Essex, and M. Davison, Competitive modes and their application, International Journal of Bifurcation and Chaos 16, 497 (2006).

19. R.A. Van Gorder and S.R. Choudhury, Classification of chaotic regimes in the $\mathrm{T}$ system by use of competitive modes, International Journal of Bifurcation and Chaos 20, 3785-3793 (2010).

20. K. Mallory and R.A. Van Gorder, Competitive modes for the detection of chaotic parameter regimes in the general chaotic bilinear system of Lorenz type, International Journal of Bifurcation and Chaos 25, 1530012 (2015).

21. G. A. Leonov, N. V. Kuznetsov, and T. N. Mokaev, Homoclinic orbits, and self-excited and hidden attractors in a Lorenz-like system describing convective fluid motion, The European Physical Journal Special Topics 224, 14211458 (2015).

22. G. A. Leonov and N. V. Kuznetsov, Hidden attractors in dynamical systems. From hidden oscillations in HilbertKolmogorov, Aizerman, and Kalman problems to hidden chaotic attractor in Chua circuits, International Journal of Bifurcation and Chaos 23, 1330002 (2013).

23. Z. Wei, W. Zhang, and M. Yao, On the periodic orbit bifurcating from one single non-hyperbolic equilibrium in a chaotic jerk system, Nonlinear Dynamics 82, 1251-1258 (2015).

24. Z. Wei, J. C. Sprott, and H. Chen, Elementary quadratic chaotic flows with a single non-hyperbolic equilibrium, Physics Letters A 379, 2184-2187 (2015). 
25. R. A. Van Gorder, Triple mode alignment in a canonical model of the blue-sky catastrophe, Nonlinear Dynamics 73, 397-403 (2013).

26. A. Tamasevicius, G. Mykolaitis, V. Pyragas, and K. Pyragas, A simple chaotic oscillator for educational purposes, European Journal of Physics 26, 61-63 (2004).

27. R. A. Van Gorder, Emergence of chaotic regimes in the generalized Lorenz canonical form: a competitive modes analysis, Nonlinear Dynamics 66, 153-160 (2011).

28. M. Itoh and L. O. Chua, Memristor oscillators, International Journal of Bifurcation and Chaos 18, 3183-3206 (2008).

29. H. A. Harrington and R. A. Van Gorder, Reduction of dimension for nonlinear dynamical systems, preprint arXiv:1508.05921 (2015). 